\title{
WHITTAKER'S WORK ON THE INTEGRAL REPRESENTATION OF HARMONIC FUNCTIONS *
}

\author{
by G. TEMPLE
}

\section{Introduction}

It is a singular honour to be invited to deliver a lecture commemorating the work of Sir Edmund Whittaker, especially before the Edinburgh Mathematical Society, whose development owes so much to his initiative and co-operation. But when I reflect on the difficulties of the task I can only exclaim in the words of St Jerome's preface to his translation of the New Testament, "Pius labor, sed periculosa praesumptio ". (10)

Sir Edmund's scientific work extended over a period of nearly sixty years and ranged over a vast field of both pure and applied mathematics, including the theory of interpolation, automorphic functions, astronomy, potential theory and the "special" functions of mathematical physics, analytical dynamics, relativity and electromagnetic theory, and quantum theory. He is also justly famous for his great scientific books and monographs, and also numerous philosophical and historical papers, including a remarkable series of twenty obituaries of distinguished physicists and mathematicians.

To attempt to summarise this great variety of work in a brief lecture would be most unsatisfactory, since it would provide only a few hurried glances at a large number of diverse topics. It seemed better therefore to select one subject as an illustration and to expound the chosen topic with reference to the influence of Sir Edmund's work.

The choice of a special subject was a matter of some difficulty, but I have decided after some hesitation to speak on Sir Edmund's investigations on harmonic functions and the "special" functions of mathematical physics from the standpoint of their representation by definite integrals.

There can be no doubt as to the permanent value of these researches. Whittaker's general solution of Laplace's equation has become an integral part of potential theory, the application of this general solution has brought a new unity into the theory of harmonic functions, and in particular has made possible the systematic investigation of Mathieu functions and Lamé functions.

Indeed the very mention of these names is an unexpected tribute to Whittaker's influence, for we owe to him not only the name of "Mathieu functions" but also the notation at present accepted for these indispensable but intractable instruments of mathematical physics.

I propose then to speak about Whittaker's work on the integral representation of harmonic functions. This alone is a vast topic which really requires the encyclopedic knowledge of the late Harry Bateman to do it full justice. My treatment will necessarily be highly selective, but I shall endeavour to

* Text of a Whittaker Memorial Lecture delivered on Friday, 17th May 1957 to the Edinburgh Mathematical Society. 
display Whittaker's work and influence in proper perspective by some brief accounts of the state of the subject both before and after the impact of Whittaker's own researches.

\section{Poisson's Integral}

The solution of partial differential equations by means of definite integrals was a favourite subject for French mathematicians in the early years of the eighteenth century, and it is well known that Ampère wrote a long paper on this topic. One of the most interesting results is Poisson's (11) solution of the wave equation

in the form

$$
\left.\begin{array}{l}
F_{t t}=a^{2}\left(F_{x x}-m F / x^{2}\right)^{*} \\
F=x^{k} \int_{0}^{\pi} \phi(x \cos \omega+a t) \sin ^{2 k-1} \omega d \omega
\end{array}\right\}
$$

where $\phi$ is an arbitrary function and $k$ a positive root of the equation

$$
k(k-1)=m \text {. }
$$

Poisson's analysis is so surprising and such an interesting anticipation of Whittaker's solution of Laplace's equation that it deserves a brief summary. To facilitate comparison with more recent work we shall transpose Poisson's solution of the wave equation into the key appropriate to the potential equation.

In Cartesian co-ordinates $x, y, z$ a harmonic function $V$ satisfies the equation

$$
V_{x x}+V_{y y}+V_{z z}=0 \text {. }
$$

In cylindrical polar co-ordinates $z, r, \phi$, defined by the equations

this equation becomes

$$
\begin{aligned}
& x=r \cos \phi, \\
& y=r \sin \phi,
\end{aligned}
$$

$$
V_{z z}+V_{r r}+r^{-1} V_{z}+r^{-2} V_{\phi \phi}=0,
$$

and, if $V$ involves the azimuthal angle $\phi$ only through a factor $\cos m \phi, \sin m \phi$, or $\exp i m \phi$, we obtain the equation

$$
V_{z z}+V_{r r}+r^{-1} V-m^{2} r^{-2} V_{\phi \phi}=0 .
$$

It is often convenient to remove the term in $V_{r}$ by means of the substitution which gives the result $\left.\begin{array}{l}V=r^{-\frac{1}{2}} U \\ U_{z z}+U_{r r}+\left(\frac{1}{4}-m^{2}\right) r^{-2} U=0,\end{array}\right\}$.

which is directly comparable with Poisson's wave equation in (2.1) on writing $z=i a t, r=x$.

Poisson's procedure is equivalent to taking as new variables $r$ and $\sigma=r+i z$.

If

we then find that

$$
U(z, r)=F(z, \sigma)
$$

$$
F_{r r}+2 F_{r \sigma}+\left(\frac{1}{4}-m^{2}\right) r^{-2} F=0 .
$$

* Here and elsewhere the suffixes $x, y, z, r, s, t, \xi, \eta, \phi, R$ denote partial derivatives. 
Poisson then examines under what conditions this equation will possess a solution of the form

$$
F=\sum r^{p+n} \phi_{n}(\sigma) ; \quad(n=0,1,2, \ldots)
$$

and he finds that

(i) $\rho$ must satisfy the indicial equation,

$$
\rho= \pm m+\frac{1}{2}
$$

(ii) $\phi_{n}(\sigma)$ must have the form

$$
\begin{aligned}
\phi_{n}(\sigma) & =B_{n} \phi^{(n)}(\sigma) / n !, \\
\text { where } & \phi^{(n)}(\sigma)=d^{n} \phi / d \sigma^{n},
\end{aligned}
$$

(iii) and the coefficients $B_{n}$ must satisfy the recurrence relation

$$
(2 \rho+n) B_{n+1}=-2(\rho+n) B_{n} .
$$

This equation Poisson solved in the form

$$
B_{n}=(-1)^{n} \int_{0}^{\pi}(1-\cos \omega)^{n}(\sin \omega)^{2 \rho-1} d \omega
$$

from which it appears that the series solution of (2.3) is nothing more than the expansion in powers of $r$ of the function

$$
\begin{aligned}
& r^{\rho} \int_{0}^{\pi} \phi\{\sigma-r(1-\cos \omega)\} \sin ^{2 \rho-1} \omega d \omega \\
= & r^{\rho} \int_{0}^{\pi} \phi(i z+r \cos \omega) \sin ^{2 \mu-1} \omega d \omega .
\end{aligned}
$$

The original equation for $V$ therefore possesses a solution of the form

$$
e^{i n \phi} r^{m} \int_{0}^{\pi} \phi(i z+r \cos \omega) \sin ^{2 m} \omega d \omega \text {. }
$$

The interest of this result, apart from the ingenuity of the analysis, is that it furnishes a general integral representation of all harmonic functions of the form $V(z, r) \exp i m \phi$, which can be expressed as a series of the type

$$
r^{m} \exp i m \phi \Sigma r^{n} \phi_{n}(r+i z) \quad(n=0,1,2, \ldots)
$$

and which satisfy a boundary condition of the form

as $r \rightarrow 0$.

$$
\exp (-i m \phi) r^{-m} V(z, r) \rightarrow f(z)=\phi(i z) \int_{0}^{\pi} \sin ^{2 m} \omega d \omega
$$

\section{Darboux's Transformation}

An elegant account of later developments of Poisson's integral by Appell and by Darboux is given by the latter in the unexpected context of his Théoric générale des surfaces (5). Darboux's investigations may be applied to yield the following method for the generalisation of particular harmonic functions. 
The elementary potential function

$$
\left(r^{2}+z^{2}\right)^{-1}
$$

yields at once a general harmonic integral

$$
\int_{a}^{b} \frac{f(t) d t}{\left\{r^{2}+(z-t)^{2}\right\}^{\frac{t}{2}}}
$$

where $f(t)$ is an arbitrary function and $a, b$ are constant limits independent of $r, z$. This integral still remains harmonic if the path of integration is taken as a closed curve in the complex $t$-plane, cut from $t=z-i r$ to $t=z+i r$. And the integral is still harmonic if the path of integration is taken along the cut itself. The substitution $t=z+i r \cos \alpha$ then gives the Poisson integral

for the case $m=0$.

$$
\int_{0}^{2 \pi} f(z+i r \cos \alpha) d a
$$

The general Poisson integral can be obtained by a sinilar argument starting with the special potential function

$$
\left(r^{2}+z^{2}\right)^{m-\frac{1}{2}} r^{-m}
$$

obtained by the inversion of $r^{-m}$.

We can also integrate along the straight line from the branch point $t=z+i \mathrm{r}$ to $t=z+i \infty$, thus obtaining, in the general case, a harmonic function of the form

$$
r^{m} e^{i m \phi} \int_{0}^{\infty} f(z+i r \cosh a) \sinh ^{2 m} a d a
$$

provided that the arbitrary function $f$ is chosen so that the integral converges.

As a matter of some interest we note finally that, when the real part of $m$ lies between $\pm \frac{1}{2}$, a second general integral can be derived from Poisson's formula (2.4) by changing the sign of $m$. When $m=0$, and the two Poisson integrals coincide, we can derive the confluent form

$$
\begin{aligned}
{\left[\frac{\partial}{\partial m} r^{m} \int_{0}^{\pi} \phi(z+i r \cos \alpha) \sin ^{2 m} \alpha d \alpha\right]_{m=0} } & \\
& =\int_{0}^{\pi} \phi(z+i r \cos \alpha) \log \left(r \sin ^{2} \alpha\right) d \alpha .
\end{aligned}
$$

\section{Donkin's Integrals}

In England the symbolical methods introduced by George Boole (3) were applied by several mathematicians, to the potential equation. Of these investigations those by Donkin (6) are especially interesting.

Donkin worked in the spherical polar co-ordinates $R, \theta, \phi$ defined by the equations

$$
z=R \cos \theta, \quad r=R \sin \theta
$$

and considered spherical harmonics of the form

$$
R^{n} F_{n}(\theta, \phi) \text {. }
$$


Writing the equation for the surface harmonic $F_{n}$ in the form

where

$$
\left\{\omega_{-n} w_{n}+n^{2}+n(n+1) \sin ^{2} \theta\right\} F_{n}+\partial^{2} F_{n} / \partial \phi^{2}=0,
$$

$$
\varpi_{n}=\sin \theta \frac{\partial}{\partial \theta}+n \cos \theta
$$

he was able to establish a recurrence relation connecting surface harmonics of orders $n-1$ and $n$ in the form

$$
\boldsymbol{F}_{n}=\varpi_{n} \boldsymbol{F}_{n-1} .
$$

Thus the general solution of (4.1) was expressed in the form

$$
F_{n}=\varpi_{n} \varpi_{n-1} \ldots \varpi_{1} F_{0}
$$

where $F_{0}$ is the general surface harmonic of order zero. Since

$$
\left(\sin \theta \frac{\partial}{\partial \theta}\right)^{2} F_{0}+\frac{\partial^{2} F}{\partial \phi^{2}}=0,
$$

$F_{0}$ was readily expressed in the form

$$
F_{0}=f\left(e^{i \phi} \tan \frac{1}{2} \theta\right) \text {. }
$$

Equation (4.2) can be modified to give the general surface harmonic of degree $n$ as

$$
F_{n}=(\sin \theta)^{-n}\left\{\sin \theta \frac{\partial}{\partial \theta} \sin \theta\right\}^{n} F_{0} .
$$

Now, in accordance with Boole's symbolic methods, the equation (4:1) for a surface harmonic $F_{n}$ of order $n$ will become the general potential equation if $n$ is replaced by the operator $R \partial / \partial R$. A similar replacement in the expression (4.3) for $F_{n}$ should therefore yield the general form of a potential function. On suitably interpreting the symbolic forms Donkin finally obtains as the general potential function

$$
f\left(\rho \sin \theta \frac{\partial}{\partial \theta} \sin \theta, e^{i \phi} \tan \frac{1}{2} \theta\right),
$$

where $\rho$ is to be replaced by $r / \sin \theta$ after the partial differentiations with respect to $\theta$ have been performed.

\section{Whittaker's Integral}

In spite of the ingenuity displayed by Poisson and Donkin in the construction of their general solutions of the potential equation, these solutions are really of limited scope and application. These restrictions are basically due to the fact that both Poisson and Donkin were primarily concerned, not with the general potential equation in three independent variables,

$$
V_{x x}+V_{y y}+V_{z z}=0
$$

but with the equations in two independent variables for potential functions of the form

or

$$
\begin{aligned}
& V=P_{m}(z, r) \exp i m \phi \\
& V=R^{n} F_{n}(\theta, \phi) .
\end{aligned}
$$


Poisson's integral (2.4),

$$
P_{m}\left(z_{1} r\right)=r^{m} \int_{0}^{\pi} \phi(z+i r \cos \alpha) \sin ^{2 n} \alpha d \alpha
$$

is curiously resistant to all attempts to generalise it to three dimensions ; and Donkin's integral (4.3),

$$
F_{n}\left(\theta_{1} \phi\right)=\sin ^{-n} \theta\left\{\sin \theta \frac{\partial}{\partial \theta} \sin \theta\right\}^{n} f\left(e^{i \phi \tan \frac{1}{2} \theta}\right)
$$

does not provide an explicit expression for a three dimensional potential.

These investigations are, I believe, good specimens of the results achieved by nineteenth century mathematicians in the search for integral representations of potential functions, and against this background Whittaker's integral stands out with special simplicity and generality $(15,16)$.

Whittaker's integral can be approached by two different paths. In the first place the elementary definite integral,

$$
\left(x^{2}+y^{2}+z^{2}\right)^{-1}=\frac{1}{2 \pi} \int_{0}^{2 \pi}(z+i x \cos \alpha+i y \sin \alpha)^{-1} d \alpha,(z>0),
$$

provides an expression for the potential of unit mass at the origin, and raises the expectation that the potential of any reasonable mass distribution can be expressed in the form

$$
\frac{1}{2 \pi} \iiint \rho(\xi, \eta, \zeta) d \xi d \eta d \zeta \int_{0}^{2 \pi}\{(z-\zeta)+i(x-\xi) \cos a+i(y-\eta) \sin a\}^{-1} d a
$$

or

$$
\int_{0}^{2 \pi} f(z+i x \cos \alpha+i y \sin \alpha, \alpha) d \alpha .
$$

On the other hand the $(2 n+1)$ terms in the Fourier series

$$
(z+i x \cos \alpha+i y \sin \alpha)^{n}=\sum_{m=-n}^{m=n} f_{n}^{m}(x, y, z) \exp i m a
$$

are each of them, like their generating function, solutions of the general potential equation. Hence any solid spherical harmonic of degree $n$ can be expressed as a linear function of the $(2 n+1)$ linearly independent functions

$$
f_{n}^{m}(x, y, z)=\frac{1}{2 \pi} \int_{0}^{2 \pi}(z+i x \cos \alpha+i y \sin \alpha)^{n} \exp (-i m \alpha) d \alpha .
$$

So much was realised by Dougall (7) in a remarkable but little known paper published in 1889-90. The crucial step taken by Whittaker was to see that any potential function which can be expanded in a series of solid spherical harmonics can be expressed in the form

$$
\Sigma C_{m, n} f_{n}^{m}=\int_{0}^{2 \pi} F(z+i x \cos a+i y \sin \alpha, a) d a,
$$

where $F$ is a periodic function of the second argument with period $2 \pi$. 
This is Whittaker's general integral representation of any potential function which is regular near the origin. It is manifest, however, that it is applicable to a far wider range of harmonic functions than those which can be expressed as power series in $x, y, z$.

\section{Legendre and Bessel Functions}

The discovery of Whittaker's integral brought a new unity into potential theory, since all harmonics must be expressible in this form. In particular the integral representation of Legendre and Bessel Functions follow as immediate consequences of Whittaker's result.

We note, in the first place, that any harmonic which is of the form

$$
V=P_{m}(z, r) \exp i m \phi
$$

must be represented by a specially simple type of Whittaker's integral. For, if

$$
\begin{aligned}
& P_{m}(z, r) \exp i m \phi=\int_{0}^{2 \pi} F\{z+i r \cos (\alpha-\phi), \alpha\} d \alpha \\
& =\int_{0}^{2 \pi} F\{z+i r \cos \beta, \phi+\beta\} d \beta
\end{aligned}
$$

then $\quad \partial^{2} F / \partial \phi^{2}=-m^{2} F$,

We thus regain an integral,

$$
e^{i m \phi} \int_{0}^{2 \pi} f(z+i r \cos \beta) e^{i m \beta} d \beta
$$

curiously reminiscent of Poisson's integral (2.4), but of a different type.

If $P_{m}(z, r) e^{i m \phi}$ has the form of a spherical harmonic,

$$
R^{n} P_{n}^{m}(\cos \theta) e^{i m \phi}, \quad(n, m \text { integral })
$$

then the arbitrary function $f$ must clearly be of the form

$$
C(z+i r \cos \beta)^{n}=C R^{n}(\cos \theta+i \sin \theta \cos \beta)^{n}
$$

and we thus obtain an integral of Laplace's type for the associated Legendre function $P_{n}^{m}(\cos \theta)$ viz.

where $C$ is a constant.

$$
P_{n}^{m}(\cos \theta)=C \int_{0}^{2 \pi}(\cos \theta+i \sin \theta \cos \beta)^{n} e^{i m \beta} d \beta
$$

Similarly, if $P_{m}(z, r)$ has the form of a cylindrical harmonic,

$$
e^{k z} J_{m}(k r) e^{i m \phi} \quad(m \text { integral }),
$$

then the arbitrary function $f$ must be of the form

$$
C \exp k(z+i r \cos \beta) \text {, }
$$

and we thus obtain an integral for the Bessel function $J_{m}$, viz.

$$
J_{m}(k r)=C \int_{0}^{2 \pi} \exp (i k r \cos \beta+i m \beta) d \beta
$$

E.M.S.-B 


\section{Mathieu Functions}

The Whittaker integral for harmonic functions was not merely of utility in unifying and simplifying a number of known results in the theory of spherical and cylindrical harmonics, but it opened up entirely new fields of research in the theory of Mathieu and Lamé functions. Indeed the systematic and fruitful investigation of periodic Mathieu functions really begins with Whittaker's proof that such functions satisfy a homogeneous integral equation with a symmetric nucleus.

This proof was first published by Whittaker in 1912 (7), but it had been given to his advanced pupils in Cambridge in 1904, and is decribed in a paper by $H$. Bateman in (1), as having been communicated to the author by Whittaker "four years ago". Meanwhile the theorem had been discovered independently by B. Sieger in 1908 (12) and by K. Aichi in the same year.

Mathieu functions occur when the potential equation is expressed in terms of $z$ and the confocal co-ordinates $\xi$, $\eta$, where

$$
x+i y=h \cosh (\xi+i \eta) \text {, }
$$

as independent variables. The equation then becomes

$$
V_{\xi \xi}+V_{\eta \eta}+h^{2}\left(\cosh ^{2} \xi-\cos ^{2} \eta\right) V_{z z}=0,
$$

and it possesses solutions of the form

$$
V=F(\xi) G(\eta) \exp (i k z / h)
$$

where the functions $F$ and $G$ satisfy the equations

$$
\text { and } \begin{aligned}
F^{\prime \prime}(\xi)-\left(A+k^{2} \cosh ^{2} \xi\right) F=0, \\
G^{\prime \prime}(\eta)+\left(A+k^{2} \cos ^{2} \eta\right) G=0 .
\end{aligned}
$$

Mathieu functions are the odd or even solutions of (7.1), which are periodic in $\eta$ with period $2 \pi$. They are eigenfunctions of the equation

where

$$
M_{\eta} G+A G=0
$$

$$
M_{\eta} \equiv \partial^{2} / \partial \eta^{2}+k^{2} \cos ^{2} \eta
$$

Now the form of the Whittaker integral (5.2) suggests that the potential function $F(\xi) G(\eta) \exp (-i k z / h)$ should be expressible in the form

$$
\int_{-\pi}^{\pi} f(Z, a) d \alpha
$$

where

$$
\begin{aligned}
Z & =z+i x \cos \alpha+i y \sin \alpha \\
& =z+i h \cosh \xi \cos \eta \cos \alpha+i h \sinh \xi \sin \eta \sin \alpha,
\end{aligned}
$$

and that the function $f(Z, a)$ should have the form

$$
f=\exp (-i k Z / h) \cdot g(\alpha)
$$

If this expectation is justified then we can conclude, on writing $\xi=0$, that the function $G(\eta)$ should be expressible in the form,

$$
G(\eta)=\int_{-\pi}^{\pi} e^{k \cos \eta \cos a} \phi(a) d a
$$


This tentative conclusion can be verified by noting that, if $G(\eta)$ is now regarded as being defined by this integral, then

$$
\begin{aligned}
M_{\eta} G(\eta) & =\int_{-\pi}^{\pi} e^{k \cos \eta \cos a} M_{a} \phi(a) \\
& -\left[\left(k \sin a \cos \eta \phi(a)+\phi^{\prime}(a)\right) e^{k \cos \eta \cos a]_{-\pi}^{\pi}} .\right.
\end{aligned}
$$

Hence if $\phi(a)$ is an even periodic solution of $M_{a} \phi+A \phi=0$, then $G(\eta)$ is an even periodic solution of $M_{\eta} G+A G=0$. But from the nature of the Mathieu equation, two periodic solutions cannot form a fundamental system, whence $\phi(\eta)=\lambda G(\eta)$, where $\lambda$ is independent of $\eta$.

It therefore follows that the even Mathieu functions satisfy the integral equation,

$$
G(\eta)=\lambda \int_{-\pi}^{\pi} e^{k \cos \eta \cos a} G(a) d a .
$$

There is a similar equation for the odd Mathieu functions.

The discovery of these integral equations for the Mathieu functions opened up a new field of research and provided simple means for constructing the functions.

\section{Lamé Functions}

The theory of Lamé functions is even more complex and difficult than the theory of Mathieu functions, and in Whittaker's original papers $(18,19)$ on the integral equation satisfied by Lamê functions, the nucleus seems to have been obtained by way of revelation rather than by reason. The key to this investigation is, however, provided by a remarkable transformation obtained by Copson thirteen years later (4) and further developed, after another fifteen years, by Erdelyi (8).

Copson's transformation enables us to pass from Cartesian co-ordinates $x, y, z$ to elliptic co-ordinates $s, t$ and the radial co-ordinate $R=\left(x^{2}+y^{2}+z^{2}\right)^{ \pm}$. It is

$$
\begin{aligned}
& x / R=\sin \theta \cos \phi=\left(i / k^{\prime}\right) \mathrm{dn} s \mathrm{dn} t, \\
& y / R=\sin \theta \sin \phi=\left(i k / k^{\prime}\right) \mathrm{cn} s \mathrm{cn} t, \\
& z / R=\cos \theta \quad=k \operatorname{sn} s \operatorname{sn} t,
\end{aligned}
$$

where $k$ is the modulus of the elliptic functions and $k^{\prime}$ is the complementary modulus $\left(i-k^{2}\right)^{\ddagger}$. The corresponding transformation of the equation for a potential function $V$ is

$$
V_{s s}-V_{t t}-k^{2}\left(\mathrm{sn}^{2} s-\mathrm{sn}^{2} t\right)\left(R^{2} V_{R R}+2 R V_{R}\right)=0 .
$$

If $V$ is a solid harmonic of degree $n$, then

$$
V=R^{n} N(s, t)
$$

and the equation for $N$ can be written in the form given by Ince (8),

where

$$
\left.\begin{array}{l}
L_{s}(N)=L_{t}(N) \\
L_{s}(N)=N_{s s}-n(n+1) k^{2} \operatorname{sn}^{2} s . N
\end{array}\right\}
$$


It is also clear that there will be potential functions of the form

$$
V=R^{n} \Lambda(s) M(t)
$$

where the functions $\Lambda$ and $M$ satisfy Lamé's equation

$A$ being a constant.

$$
\begin{aligned}
& L_{s}(\Lambda)=A \Lambda, \\
& L_{t}(M)=A M,
\end{aligned}
$$

The form of Whittaker's integral equation for Mathieu functions (9.2) now suggests an examination of integral equations of the form

$$
\mu(s)=\lambda \int_{0}^{4 K} N(s, t) \mu(t) d t
$$

where $4 K$ is the period of the elliptic functions introduced above, and the nucleus $N(s, t)$ is a surface harmonic of order $n$ satisfying equation (8.1). A simple argument, analogous to that employed for Mathieu functions, then shows that this equation (8.2) is satisfied by Lamé functions of period $4 K$.

A suitable form for the nucleus is the biaxial harmonic

where

$$
N(s, t)=P_{n}(\mu)
$$

$$
\begin{gathered}
\mu=\cos \theta \cos \theta_{0}+\sin \theta \sin \theta_{0} \cos \left(\phi-\phi_{0}\right) \\
=k^{2} \operatorname{sn} s \operatorname{sn} t \operatorname{sn} s_{0} \operatorname{sn} t_{0} \\
\quad+\left(i / k^{\prime}\right)^{2} \mathrm{dn} s \operatorname{dn} t \operatorname{dn} s_{0} \operatorname{dn} t_{0} \\
\quad-\left(k / k^{\prime}\right)^{2} \operatorname{cn} s \text { en } t \text { en } s_{0} \text { en } t_{0}
\end{gathered}
$$

where $\theta_{0}, \phi_{0}, s_{0}, t_{0}$ are arbitrary parameters. The nucleus first discovered by Whittaker is obtained by writing

when

$$
s_{0}=K, t_{0}=K+i K^{\prime} \text {, }
$$

$$
P_{n}(\mu)=P_{n}(k \operatorname{sn} s \sin t) .
$$

As shown by Copson (4) other simple nuclei are

$$
\begin{array}{ll}
P_{n}\left(i k / k^{\prime} . \operatorname{cn} s \text { cn } t\right), & \left(s_{0}=0, t_{0}=K+i K^{\prime}\right) \\
P_{n}\left(i / k^{\prime} . \quad \operatorname{dn} s \operatorname{dn} t\right), & \left(s_{0}=0, t_{0}=K\right) .
\end{array}
$$

We may also take the nucleus in the form (18)

$$
N(s, t)=(z+i x \cos \eta+i y \sin \eta)^{n} / R^{n}
$$

(where $\eta$ is arbitrary) and thus obtain an integral representation in the standard form of Whittaker's general solution of the potential equation (5.2).

In fact Whittaker's original discovery of the integral representation of Lamé functions opened the way for numerous interesting investigations, many of which he subsequently described in later editions of Modern Analysis (Chapter XXIII), and gave a new vitality to research in this difficult and obscure subject.

\section{Integral Operators}

In describing Whittaker's investigations on the integral representation of Lamé functions it was impossible to avoid frequent reference to the work of his students and colleagues. In this section and the next I propose to refer 
briefly to certain more recent developments in the theory of the integral representation of potential functions, in order to illustrate the fruitfulness of this method and the extent of Whittaker's direct or indirect influence.

Whittaker's fundamental formula,

written in the form

$$
V=\int_{0}^{2 \pi} f(z+i x \cos \alpha+i y \sin \alpha) d a
$$

where

$$
V=\oint f(u, \zeta) d \zeta / \zeta
$$

$$
\begin{aligned}
& u=z+\zeta \sigma+\zeta^{-1} \sigma^{*}, \\
& \sigma=\frac{1}{2}(y+i x), \quad \sigma^{*}=\frac{1}{2}(y-i x) \\
& \zeta=i e^{-i a},
\end{aligned}
$$

forms the starting point of the "method of integral operators" as developed by Stefan Bergman (2). This method can be used to develop the properties of harmonic functions in three dimensions in terms of the properties of analytic functions of two complex variables $f(u, \xi)$. It can also be extended to provide a representation of harmonic vectors

in the form

$$
\mathbf{H}=(P, Q, R)
$$

$$
P=\int_{0}^{2, \pi} i \cos a f d a, Q=\int_{0}^{2 \pi} i \sin a f d a, R=\int_{0}^{2 \pi} f d \alpha
$$

where, as before, $f$ is a function of $Z=z+i x \cos a+i y \sin a$ and $a$. These vectors satisfy the equations

$$
\operatorname{div} \mathbf{H}=0, \quad \text { and } \quad \operatorname{curl} \mathbf{H}=\mathbf{0} .
$$

If we choose $f(\mu, \xi)$ to be the quotient of two polynomials in $u$ and $\zeta$, we obtain a class of functions which includes all algebraic harmonic functions. These functions are defined on certain three dimensional analogues of Rieman surfaces, and it is possible to obtain generalisations of the Rieman-Roch theorem and of Abel's theorem.

\section{Generalised Axially Symmetric Potential Theory}

The potential equation in $(p+2)$ dimensions is

$$
\Sigma \partial^{2} V / \partial x_{k}^{2}=0, \quad(k=1,2, \ldots, p+2),
$$

and the axially symmetric solutions, which depend only on the variables $x=x_{1}$, and $g=\left(x_{2}^{2}+x_{3}^{3}+\ldots+x_{p+2}^{2}\right)^{\frac{1}{t}}$, satisfy the equation

or

$$
\left.\frac{\partial}{\partial x}\left\{y^{p} \frac{\partial \phi}{\partial x}\right\}+\frac{\partial}{\partial y}\left\{y^{p} \frac{\partial \phi}{\partial y}\right\}=0\right\} \text {. }
$$

The general theory of this equation has been widely developed by Weinstein and his colleagues $(13,14)$ the restriction to integral values of $p$ being removed. The "generalised axially symmetric potentials" which satisfy this equation E.M.S.-B 2 
have numerous applications and an abundant literature now flourishes on this subject.

Among the many interesting developments is the theory of Tricomi's equation

$$
\eta f_{\xi \xi}+f_{\eta \eta}=0
$$

which is of fundamental importance in the theory of transonic flow. One of the basic problems is the determination of a fundamental integral which is defined in the entire plane of $\xi$ and $\eta$ and has a logarithmic singularity at an arbitrary point $(0, b)$.

Weinstein's solution (13) depends upon the reduction of the Tricomi equation by the transformation

which leads to the equation

$$
x=\xi, y=\frac{2}{3} \eta^{\frac{3}{2}}
$$

$$
f_{x x}+f_{y y}+(1 / 3 y) f_{y}=0
$$

for an axially symmetric potential in $2 \frac{1}{3}$ dimensions. The fundamental solution has the form

$$
f=u(x, y) \log \left\{x^{2}+(y-a)^{2}\right\}+v(x, y),
$$

where $u$ and $v$ are regular and analytic at the singular point. In space of 3 dimensions such a solution would correspond to the potential of a uniform ring $x=0, y=a$, and would reduce to $\left(x^{2}+a^{2}\right)^{-\frac{1}{2}}$ on the axis. This suggests that in space of $2 \frac{1}{3}$ dimensions the fundamental solution should reduce to $\left(x^{2}+a^{2}\right)^{-p / 2}$ where $p=2 \frac{1}{3}-2$. Poisson's integral (2.4) then furnishes the solution in the form

$$
\begin{aligned}
f & =C \int_{0}^{\pi}\left\{(x+i y \cos a)^{2}+a^{2}\right\}^{-\frac{1}{8}} \sin ^{-\frac{2}{8}} \alpha d a \\
& =C \int_{0}^{\pi}\left\{\left(\xi+\frac{2}{3} i \eta^{\frac{3}{2}} \cos \alpha\right)^{2}+\frac{4}{5} b^{3}\right\}^{-\frac{1}{6}} \sin ^{-\frac{2}{3}} a d \alpha
\end{aligned}
$$

where the singular point is $\xi=0, \eta=b$.

\section{Unsolved Questions}

The fertility of Whittaker's potential integral (5.2) is by no means exhausted by the copious developments and applications which have been made in recent years, and a number of interesting problems still await solution. So far as the regular potential functions are concerned Whittaker's theorem shows that an integral representation is always possible in the form

$$
\int_{0}^{2 \pi} f(z+i x \cos \alpha+i y \sin \alpha, \alpha) d \alpha
$$

and this result undoubtedly serves to unify the theory of those special functions (such as $P_{n}^{m}$ and $J_{m}$ ) which are regular. But, as Whittaker himself pointed out, there are other potential functions (such as $Q_{0}$ ), which are not regular, and which can also be represented in the same form. An examination of the representation of irregular functions by Whittaker's integral would lead to 


\section{WHITTAKER'S WORK ON HARMONIC FUNCTIONS}

interesting results, and should serve to unify the theory of the "second solutions " (such as $Q_{n}^{m}$ and $Y_{n}$ ) of the potential equations.

Another problem which has not been clarified is the precise relation between integrals of Whittaker's type

of Poisson's type

$$
\int_{0}^{2 \pi} f(z+i r \cos a) e^{i m a} d a
$$

and of Heine's type

$$
r^{m} e^{i m \phi} \int_{0}^{2 \pi} g(z+i r \cos a) \sin ^{2 m} a d a
$$

$$
\int_{0}^{\infty} h(z+i z \cosh \beta) e^{i m \beta} d \beta
$$

each of which can represent an axially symmetric potential.

Finally it may be worth while to examine integrals of Poisson's type for the case $m<-1$ by employing Hadamard's theory of the finite part of divergent integrals, and by correlating the results with the recursion formulæ so effectively employed by Weinstein (14).

\section{Conclusion}

I hope that I have now justified my selection of the subject of Whittaker's investigations on the integral representation of potential functions on the topic of this memorial lecture. For my part it has been a most rewarding study and $I$ have gained an even deeper appreciation of the peculiar genius of Sir Edmund Whittaker ; and, in particular, of the simplicity, comprehensiveness and fertility which characterise these researches.

The starting point was the simple observation that all the solid spherical harmonics of order $n$ are given by the Fourier coefficients of the basic potential function

$$
(z+i x \cos a+i y \sin a)^{n} .
$$

This apparently trivial result not only gave a new principle of order in the theory of Legendre functions but also issued in a general form for the integral representation of all regular potential functions. It gave a new impetus to the study of Mathieu functions and initiated a new attack on the theory of Lamé functions. Even now the full consequences of Whittaker's work are far from being fully explored, and we may confidently expect many new and fruitful applications to enlighten and enliven the study of the special functions of mathematical physics.

\section{REFERENCES}

(1) H. Bateman, Trans. Camb. Phil. Soc., 21 (1912), 193.

(2) S. Bergman, Trans. Amer. Math. Soc., 59 (1946), $216-247$; Duke Math. Journ., 13 (1946), 419.459.

(3) G. Boole, Phil. Trans. R.S. (1844), 225-282.

(4) E. T. Copson, Proc. Edin. Math. Soc., (ii) 1 (1927), 62-64. 
(5) G. Darboux, Leçons sur la théorie générale des Surfaces, $2^{e}$ Partie (1889), chap. III, 54-70.

(6) W. F. Donkin, Phil. Trans. R.S. (1857), 43-58.

(7) J. Dougall, Proc. Edin. Math. Soc., 8 (1889-90), 86-89.

(8) A. Erdelyi, Proc. Edin. Math. Soc. (ii), 7 (1942), 3-15.

(9) E. L. Ince, Proc. Roy. Soc. Edin., 60 (1939-40), 47-63.

(10) St Jerome, Epistula ad Damasum.

(11) S. D. Poisson, Journ. $d$ l'École Royale Polytechnique, tome XII, cahier XIX, (1823), 215-248.

(12) S. Sieger, Ann. d. Phys., 27 (1908), 646.

(13) A. Weinstein, Bull. Amer. Math. Soc., 59 (1953), 20-38.

(14) A. Weinstein, Proc. Int. Congress of Math. (1954), 3, 264-269.

(15) E. T. Whittaker, M.N.R.A.S., 62 (1902), 617-620.

(16) E. T. Whittaker, Math. Ann., 57 (1903), 333-355.

(17) E. T. Whittaker, Proc. V. Int. Congress of Math. (1912), 1, 366-371.

(18) E. T. Whittaker, Proc. Roy. Soc. Edin., 32 (1914), 75-80.

(19) E. T. Whittaker, Proc. Lond. Math. Soc. (ii), 14 (1914), 260-268.

\section{The Mathematical Institute}

10 Parks Road

OXFord 